\title{
Predictive Factors for Persistent Pain and Poor Recovery of Health Status 1 Year after Whiplash Injury (Quebec Grade 1 and 2): Results from the ESPARR Cohort
}

\author{
Inès Khati ${ }^{1,2}$, Laetitia Chossegros ${ }^{1,2}$, Pierrette Charnay1,2, Hélène Tardy ${ }^{1,2}$, \\ Anne-Laure Perrine1-3, Bernard Laumon ${ }^{1,2}$, Martine Hours ${ }^{1,2}$ \\ ${ }^{1}$ Unité Mixte de Recherche Epidémiologique et de Surveillance Transport Travail Environnement (UMRESTTE) \\ $\mathrm{N}^{\circ}$ T9405: Université de Lyon, Lyon, France \\ ${ }^{2}$ IFSTTAR, UMRESTTE, Bron, France \\ ${ }^{3}$ Institut National de Veille Sanitaire, Saint Maurice, France \\ Email: martine.hours@ifsttar.fr
}

Received 13 January 2014; revised 21 February 2014; accepted 19 March 2014

Copyright (C) 2014 by authors and Scientific Research Publishing Inc.

This work is licensed under the Creative Commons Attribution International License (CC BY).

http://creativecommons.org/licenses/by/4.0/

(c) (i) Open Access

\begin{abstract}
Background: A large number of studies have addressed whiplash injury, and many meta-analyses have sought to highlight chronicity factors; the implicated processes, however, remain a matter of debate. The present study used data from the ESPARR cohort (an on-going prospective study of a representative cohort of road accident victims in the Rhône administrative département of France). The objectives were to describe the consequences of whiplash injury and to determine prognostic factors for poor recovery and persistent pain at 1 year post-accident. Methods: The cohort included 255 "pure" whiplash victims, 173 of whom responded to the 1-year follow-up questionnaire. Correlations between explanatory variables and health and pain status were explored by modified Poisson regression to provide adjusted relative risk (RR) values. Results: Half of the victims had not fully recovered health status by 1 year. The main factor associated with nonrecovery was pain $(R R=1.3 ; 1.0-1.7)$. $A$ birth in the family preceding the accident emerged as another factor $(R R=1.5 ; 1.2-1.9)$. Victims responsible for their accident were twice as likely to report being free of pain as those not responsible $(R R=0.5 ; 0.3-0.8)$. No correlation emerged with accident-related characteristics or PTSD. Conclusions: The present results extend our understanding of whiplash injury. Residual pain is the fundamental factor causing whiplash victims to feel that they have not recovered good health. Our findings suggest this may be bound up with physical factors (gender susceptibility); external factors such as having to carry weights (such as a baby) and with perceiving oneself as a victim are not incompatible with this hypothesis.
\end{abstract}




\section{Keywords}

\section{Whiplash, One Year-Follow-up, Recovered Health, Pain, Predictive Factor}

\section{Introduction}

Whiplash is a neck trauma caused by sudden acceleration/deceleration, usually due to a crash into the rear of the victim's road vehicle [1]: the head being flung forward then immediately backward induces hyperflexion followed by hyperextension of the cervical spine at the moment of impact. It is the most frequent injury sustained in road accidents. While generally graded as a minor lesion (AIS1) on the Abbreviated Injury Scale (AIS) [2], it may nevertheless cause lasting disability. Many studies have reported a risk of chronicization of symptoms, defined by the Quebec Task Force as residual pain persisting 6 months or more after the accident, impairing neck mobility and everyday activity [1]; this may affect up to $74 \%$ of victims 1 or even 2 years post-accident [3].

A very large number of studies have addressed whiplash injury, and many meta-analyses have sought to identify chronicity factors [4]-[9]. Long-term consequences are now known to depend on primary lesion parameters and also on sociodemographic and psycho-behavioral factors [7] [10], but the precise processes involved remain a matter of debate [5]. Relevant clinical evidence, other than symptomatology, is lacking, and the impact of whiplash injury needs to be analyzed, especially in France, where there have been few dedicated studies. Discerning the factors of chronicization should help identify at-risk subjects and optimize management. The present study therefore focused on risk factors that may be implicated in persistent whiplash symptomatology, with particular attention to pain as an indicator: it is a decisive factor in evaluating cervical distortion, as it is frequently the presenting complaint [11].

The present study used data from the ESPARR (Etude et Suivi d'une Population d'Accidentés de la Route dans le Rhône) study of a representative cohort of road accident victims in the Rhône administrative département of France, followed up for 5 years after their accident [12]. The objectives were to describe the consequences of whiplash injury and to determine prognostic factors for poor recovery and persistent pain at 1 year post-accident.

\section{Material and Methods}

\subsection{The ESPARR Cohort}

The inclusion period lasted from October 2004 to December 2005, with the cooperation of all of the emergency and intensive care units of the Rhône department (France). After an initial assessment at the time of the accident, patients were offered follow-up at 6 months and 1, 2, 3 and 5 years. Further details on recruitment methodology are to be found in a previous report [12]. The ESPARR cohort constitutes a subpopulation that has been shown to be representative of the exhaustive population of a Registry (Registre des Victimes d'Accidents de la Circulation Routière du Rhône: Rhône Road Traffic Accident Victims Registry) which has recorded all road-accident victims who consulted in or were admitted to any of the hospital departments in the area since 1995 [12]-[14].

The cohort comprises 1168 adults (aged 16 years or over). At inclusion, an experienced physician coded all lesions according to the AIS criteria [2], working from the initial medical records, which covered symptomatology, clinical and biological examination results, and imaging where judged necessary. Each elementary lesion was thus coded, as was severity on a scale from 1 (minor) to 6 (maximal). After the patients (or their family) had provided consent, an initial questionnaire was filled out and was then systematically followed up at 6 months and 1, 2, 3 and 5 years by postal self-administration.

\subsection{Study Population}

The present study selected victims who had sustained "pure" whiplash injury, to the exclusion of other cervical spine lesions graded AIS $\geq 3$ or whiplash injuries (AIS $<3$ ) associated with 1 or more AIS $\geq 2$ lesions in a dif- 
ferent body region. The ESPARR cohort included 255 such victims, 173 of whom (68\%) responded to the questionnaire. Initial AIS grades were compared to Quebec Task Force categories, a standard classification founded on interview-based diagnosis, clinical findings and X-ray [1], universally employed for whiplash. It comprises 4 cervical distortion severity grades: grade 1 , simple contusion; grade 2 , neck sprain; grade 3 , cervical symptomatology with associated neurological abnormality (such as impaired tendon reflex or motor and/or sensory impairment); and grade 4, clinical signs of major structural pathology involving severe osteoarticular lesions (fracture or dislocation). Concretely, the consultation reports of 40 ESPARR subjects were reviewed and classified on the Quebec system. Comparing the two classification methods (AIS and Quebec) found perfect agreement in all 40 cases, allowing transposition between the two.

Finally, all subjects with lesions classified as cervical contusion (AIS code $310402=$ grade 1 ) or neck sprain (AIS code 640278 = grade 2) were included. Cervical spine lesions with AIS code 640278 but with associated neurologic abnormality ( $\mathrm{n}=2$; grade 3 ), on the other hand, were excluded, bringing the number of subjects included to 171, comprising 62 grade- 1 and 109 grade-2 victims.

In what follows, distributions are reported in terms of the Quebec classification.

\subsection{Variables and Measurement Tools}

The 2 principal assessment criteria were: incomplete recovery of health and persistent pain at 1 year. The former was assessed by a questionnaire item on medical health status at 1 year; subjects reporting complete recovery were compared with those reporting partial or no recovery (i.e., responses "Health status improved but not fully recovered", "Health status stable" or "Health status worsened"). The latter was based on an item from the World Health Organization Quality of Life tool (WHOQOL-bref) [15]: "Does physical pain prevent you from doing what you need to do?”; subjects checking the responses "A little”, “A moderate amount”, "Very much" or "An extreme amount" were compared with those responding "Not at all".

For analysis, the explanatory (predictive and associated) variables were:

- socio demographic factors: gender, age, family situation, educational level;

- life event factors during the year preceding the accident: hospital admission or death of a relative, birth, break-up of the couple, money problems;

- pre-accident psychological history (sleep disorder, use of anti-depressants/anxiolytics, psychological care);

- accident-related factors: type of road-user, reason for travel, position in vehicle, antagonist, impact direction, responsibility in accident, intention to lodge a complaint, presence of an injured friend or family member;

- factors relating to the lesion and immediate consequences: whiplash grade, time off work;

- factors relating to consequences at 1 year: post-traumatic stress disorder (PTSD, assessed on the PTSD Checklist Scale (PCLS), with score $\geq 44$ indicating probable PTSD [16] [17]), disabling physical pain, disturbed leisure, disturbed work, financial impact, head pain, "feeling low", vertigo, memory disorder, sensitivity to noise, sensitivity to light.

\subsection{Statistics}

The representativeness of the study population was assessed by comparing respondents and non-respondents at the 1-year follow-up by chi² (significance threshold, 5\%), or Fisher's exact test where samples were too small. Likewise, ESPARR cohort whiplash victims were compared with the exhaustive Rhône Registry of whiplash victims, on the same tests.

Correlations between explanatory variables and health status or pain were explored by modified Poisson regression to provide adjusted relative risk (RR) values. Variables associated with the dependent variable on univariate analysis (with a $20 \%$ threshold) were included in a full model, and a descending procedure progressively eliminated non-significant factors.

For multivariate analysis, 2 models were constructed for each independent variable: one including (predictive) factors inventoried at the time of the accident, and the other also including factors inventoried at 1 year.

In all models, age, gender and whiplash grade were included, as adjustment variables independently of significance level. Accident-related variables were initially included in the full model systematically, and withdrawn when non-significant.

Analysis was performed on the "proc genmod" procedure of the SAS 9.2 software package. 


\section{Results}

\subsection{Description of Population}

\subsubsection{Representativeness of the Population}

One hundred and seventy one of the 253 subjects (67.6\%) responded at 1 year, with no significant differences between respondents and non-respondents on inclusion criteria or for accident-related factors. Nor did the study population differ from the road-accident Registry population in terms of age, gender, whiplash severity, type of road-user or reason for travel.

\subsubsection{Sociodemographic Data and Accident Characteristics}

The accident caused grade-2 cervical lesions in two-thirds of victims (Table 1). Victims were basically young

Table 1. Comparison of sociodemographic and personal characteristics, in whiplash victims having recovered versus not recovered initial health status $(n=171)$.

\begin{tabular}{|c|c|c|c|c|c|c|c|c|}
\hline & & \multicolumn{2}{|c|}{ Non-recovery $(\mathrm{n}=95)$} & \multicolumn{2}{|c|}{ Recovery $(\mathrm{n}=76)$} & \multicolumn{2}{|c|}{ Total $(\mathrm{n}=171)$} & \multirow[b]{2}{*}{ p-value } \\
\hline & & $\mathrm{n}$ & $\%$ & $\mathrm{n}$ & $\%$ & $\mathrm{n}$ & $\%$ & \\
\hline \multicolumn{9}{|l|}{ Whiplash grade } \\
\hline & grade 1 & 32 & 33.7 & 30 & 39.5 & 62 & 36.3 & ns \\
\hline & grade 2 & 63 & 66.3 & 46 & 60.5 & 109 & 63.7 & \\
\hline \multicolumn{9}{|c|}{ Sociodemographic data } \\
\hline \multicolumn{9}{|l|}{ Gender } \\
\hline & Female & 65 & 68.4 & 42 & 55.3 & 107 & 62.6 & ns \\
\hline & Male & 30 & 31.6 & 34 & 44.7 & 64 & 37.4 & \\
\hline \multicolumn{9}{|l|}{ Age } \\
\hline & $16-24$ yrs & 22 & 23.2 & 32 & 42.1 & 54 & 31.6 & $<0.05$ \\
\hline & $25-34$ yrs & 32 & 33.7 & 14 & 18.4 & 46 & 26.9 & \\
\hline & $35-44$ yrs & 25 & 26.3 & 15 & 19.7 & 40 & 23.4 & \\
\hline & $>=45 \mathrm{yrs}$ & 16 & 16.8 & 15 & 19.7 & 31 & 18.1 & \\
\hline Family situation & & & & & & & & ns \\
\hline & Single, divorced, widowed & 44 & 46.3 & 40 & 52.6 & 84 & 49.1 & \\
\hline & In couple & 51 & 53.7 & 36 & 47.4 & 87 & 50.9 & \\
\hline Educational level & & & & & & & & ns \\
\hline & Secondary & 31 & 32.6 & 24 & 31.6 & 55 & 32.2 & \\
\hline & Higher & 64 & 67.4 & 52 & 68.4 & 116 & 67.8 & \\
\hline \multicolumn{9}{|c|}{ Pre-accident events } \\
\hline \multicolumn{2}{|c|}{ Psychological history } & & & & & & & ns \\
\hline & No & 65 & 68.4 & 55 & 72.4 & 120 & 70.2 & \\
\hline & Yes & 30 & 31.6 & 21 & 27.6 & 51 & 29.8 & \\
\hline \multicolumn{2}{|l|}{ Birth, Adoption } & & & & & & & $<0.05$ \\
\hline & No & 81 & 85.3 & 74 & 97.4 & 155 & 90.6 & \\
\hline & Yes & 14 & 14.7 & 2 & 2.6 & 16 & 9.4 & \\
\hline \multicolumn{2}{|c|}{ Death, hospital admission } & & & & & & & $<0.05$ \\
\hline & No & 56 & 58.9 & 56 & 73.7 & 112 & 65.5 & \\
\hline & Yes & 39 & 41.1 & 20 & 26.3 & 59 & 34.5 & \\
\hline \multirow[t]{3}{*}{ Break-up } & & & & & & & & ns \\
\hline & No & 79 & 83.2 & 65 & 85.5 & 144 & 84.2 & \\
\hline & Yes & 16 & 16.8 & 11 & 14.5 & 27 & 15.8 & \\
\hline
\end{tabular}

ns: non-significant $\mathrm{p}>0.05$. 
and female: almost two-thirds under 35 years of age, and 63\% women. The predominant accident-related characteristics were: driver (84\%), 4-wheel vehicle user (87\%), and crash with another motor vehicle (82\%). 33\% of impacts were to the rear of the vehicle, and respectively 22\% and 27\% Frontal or lateral (Table 2).

Table 2. Comparison of accident conditions and other accident-related factors in whiplash victims having recovered versus not recovered initial health status $(n=171)$.

\begin{tabular}{|c|c|c|c|c|c|c|c|}
\hline & \multicolumn{2}{|c|}{ Non-recovery $(n=95)$} & \multicolumn{2}{|c|}{ Recovery $(n=76)$} & \multicolumn{2}{|c|}{ Total $(n=171)$} & \\
\hline & $\mathrm{n}$ & $\%$ & $\mathrm{n}$ & $\%$ & $\mathrm{n}$ & $\%$ & \\
\hline \multicolumn{8}{|l|}{ Accident conditions } \\
\hline \multicolumn{8}{|l|}{ Type of road-user } \\
\hline Pedestrian, bicycle, blades, skates & 4 & 4.2 & 5 & 6.6 & 9 & 5.3 & \\
\hline 2-wheel motor & 6 & 6.3 & 7 & 9.2 & 13 & 7.6 & ns \\
\hline 4-wheel motor & 85 & 89.5 & 64 & 84.2 & 149 & 87.1 & \\
\hline \multicolumn{8}{|l|}{ Reason for travel } \\
\hline Trip to work/school & 37 & 38.9 & 30 & 39.5 & 67 & 39.2 & \\
\hline Work purposes & 5 & 5.3 & 2 & 2.6 & 7 & 4.1 & ns \\
\hline Other & 53 & 55.8 & 44 & 57.9 & 97 & 56.7 & \\
\hline \multicolumn{8}{|l|}{ Position in vehicle } \\
\hline Driver & 77 & 81.1 & 67 & 88.2 & 144 & 84.2 & \\
\hline Passenger & 18 & 18.9 & 9 & 11.8 & 27 & 15.8 & ns \\
\hline \multicolumn{8}{|l|}{ Impact direction } \\
\hline Frontal & 23 & 24.2 & 15 & 19.7 & 38 & 22.2 & \\
\hline Rear & 35 & 36.8 & 22 & 28.9 & 57 & 33.3 & ns \\
\hline Lateral & 24 & 25.3 & 22 & 28.9 & 46 & 26.9 & \\
\hline Don’t know/no reply & 13 & 13.7 & 17 & 22.4 & 30 & 17.5 & \\
\hline \multicolumn{8}{|l|}{ Antagonist } \\
\hline None & 4 & 4.2 & 12 & 15.8 & 16 & 9.4 & \\
\hline Other (pedestrian, fixed obstacle...) & 6 & 6.3 & 9 & 11.8 & 15 & 8.8 & 0.01 \\
\hline Motor vehicle & 85 & 89.5 & 55 & 72.4 & 140 & 81.9 & \\
\hline \multicolumn{8}{|c|}{ Other factors directly related to the accident } \\
\hline \multicolumn{8}{|l|}{ Sick leave following accident } \\
\hline No & 5 & 5.3 & 13 & 17.1 & 18 & 10.5 & 0.01 \\
\hline Yes & 62 & 65.3 & 36 & 47.4 & 98 & 57.3 & \\
\hline Don’t know/no reply & 28 & 29.5 & 27 & 35.5 & 55 & 32.2 & \\
\hline \multicolumn{8}{|l|}{ Responsibility in accident } \\
\hline No & 79 & 83.2 & 49 & 64.5 & 128 & 74.9 & $<0.01$ \\
\hline Yes & 16 & 16.8 & 27 & 35.5 & 43 & 25.1 & \\
\hline \multicolumn{8}{|l|}{ Intention to lodge complaint } \\
\hline No & 52 & 54.7 & 52 & 68.4 & 104 & 60.8 & $<0.05$ \\
\hline Yes & 16 & 16.8 & 4 & 5.3 & 20 & 11.7 & \\
\hline Don’t know/no reply & 27 & 28.4 & 20 & 26.3 & 47 & 27.5 & \\
\hline \multicolumn{8}{|c|}{ Friend or family member involved } \\
\hline No & 65 & 68.4 & 56 & 73.7 & 121 & 70.8 & ns \\
\hline Yes & 30 & 31.6 & 20 & 26.3 & 50 & 29.2 & \\
\hline
\end{tabular}


Likewise, the grade- 1 and grade-2 whiplash subgroups showed no significant differences except in type of road-user $(\mathrm{p}=0.01)$ and type of antagonist $(\mathrm{p}=0.05)$ : $82 \%$ of grade- 1 victims used 4 -wheel vehicles and the same percentage crashed with another motor vehicle, while only $37 \%$ of grade- 2 victims were in 4 -wheel vehicles and $52 \%$ crashed with another motor vehicle (32\% of grade- 2 victims having no antagonist).

Only a minority (25\%) of subjects were responsible for the crash. At the time of the accident, only $12 \%$ intended to lodge a complaint. Like in the ESPARR cohort as a whole, $57 \%$ of whiplash victims had sick leave immediately after the accident.

$57.8 \%$ of grade- 2 and $51.6 \%$ of grade- 1 victims (non-significant difference) had not recovered their health status at 1 year.

\subsection{Accident Consequences at 1 Year}

\subsubsection{Health Status}

One year post-accident, more than half of the victims reported health status as not being back to normal (Table 3); half likewise considered their physical pain to be disabling in daily life and a quarter said they needed medical care to cope. Seventy-nine percent of subjects claiming not to have recovered good health reported disabling pain at 1 year, compared to $13 \%$ of those claiming full recovery.

Those who had not recovered good health were more likely to report persistent problems: notably, headache ( $48 \%$ vs. $5 \%$ ), feelings of vertigo ( $34 \%$ vs. $7 \%$ ), memory disorder (34\% vs. $4 \%$ ) or sensitivity to noise (33\% vs. $4 \%)$.

\subsubsection{Impact on Daily Life and Mood}

Regarding daily-life impact: financial consequences and disturbed leisure affected a quarter of victims; work was affected in $30 \%$ of cases (Table 3). Emotionally, there was a tendency to "feel low" or have negative feelings; $14.6 \%$ developed PTSD (6.6\% of those who recovered good health and 21.1\% of those who did not). 30\% of those who failed to recover good health reported frequent negative feelings (vs. 13\%).

\subsection{Predictive and Associated Factors for Non-Recovery of Health at 1 Year}

In the first model, including pre-accident and accident-related factors, whiplash grade was independent of health recovery at 1 year. In contrast, female gender $(R R=1.3 ; 1.0-1.8)$, age $25-34$ years $(R R=1.6 ; 1.1-2.3)$, a birth during the previous year $(\mathrm{RR}=1.6 ; 1.2-2.1)$, a death or hospitalization in the family during the previous year $(\mathrm{RR}=1.3 ; 1.0-1.7)$ or non-responsibility in the accident $\left(\mathrm{OR}_{\text {non-responsible }}=1.4 ; 1.0-2.5\right]$ (Table 4$)$ were associated with higher risk of non-recovery. There was no interaction between gender and birth in the family during the previous year.

In the second model, also including consequences observed at 1year, non-recovery was no longer associated with a birth during the previous year, responsibility for the accident or death of a relative during the previous year, but was associated with memory disorder and, above all, pain.

PTSD at 1 year, non-responsibility for the accident and intention to lodge a complaint were significantly associated with non-recovery on univariate analysis but not after adjustment on the other variables. No correlation emerged between recovery of health status and psychological history (depression, anxiety, sleep disorder, psychological care) or crash characteristics.

\subsection{Factors Associated with a Disabling Pain}

As pain was a factor strongly associated with non-recovery of health status, predictive factors for pain were explored.

Victims with grade-2 whiplash tended more often to have residual pain at 1 year, although the difference was non-significant. Women showed a 30\% greater risk than men. Victims responsible for their accident were twice as likely to be free of pain as those who were not responsible ( $R R=0.5 ; 0.3-0.9)$. Finally, a birth during the year before the accident was also associated with elevated risk of pain ( $R R=1.6 ; 1.2$ - 2.1) (Table 5).

In the second model, pain was associated with consumption of medication, disturbed leisure and disturbed work.

No correlation emerged with accident-related characteristics (passenger vs. driver, involvement of friend or 
Table 3. Comparison of consequences at 1 year between whiplash victims having recovered versus not recovered initial health status.

Non-recovery $(n=95)$ Recovery $(n=76)$ Total $(n=171)$

西

$\mathrm{n}$

$\%$

n $\quad \% \quad p$

Physical health status

Disabling physical pain

$\begin{array}{llllllll}\text { No } & 19 & 20.0 & 66 & 86.8 & 85 & 49.7 & <0.0001 \\ \text { Yes } & 76 & 80.0 & 10 & 13.2 & 86 & 50.3 & \end{array}$

\section{Headache}

Not at all or Not more than usual

A little more or Much more than usual

72

94.7

48.4

4

$5.3 \quad 50$

29.2

\section{Vertigo}

Not at all or Not more than usual

A little more or Much more than usual

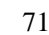

93.4

33.7

5

6.6

$78.4<0.0001$

Need for medical treatment

$$
\text { No }
$$

Sensitivity and problems

\section{Sensitivity to noise}

Not at all or Not more than usual

A little more or Much more than usual

\section{Sensitivity to light}

Not at all or Not more than usual

A little more or Much more than usual

\section{Memory disorder}

Not at all or Not more than usual

A little more or Much more than usual

Sleep disorder

$$
\text { Not at all }
$$

A little, sometimes

Often, very often

Difficulty concentrating

Not at all

A little, sometimes

Often, very often
67.4

9

75

\section{7}

1.3

22

12.9

39

$\begin{array}{lllll}73 & 96.1 & 136 & 79.5 & <0.0001 \\ 3 & 3.9 & 35 & 20.5 & \\ & & & & \\ 53 & 69.7 & 92 & 53.8 & <0.001 \\ 15 & 19.7 & 45 & 26.3 & \\ 8 & 10.5 & 34 & 19.9 & \end{array}$




\section{Continued}

Consequences in daily life

Financial consequences at 1 year

\begin{tabular}{|c|c|c|c|c|c|c|c|}
\hline & & & & & & & \\
\hline No & 62 & 65.3 & 67 & 88.2 & 129 & 75.4 & $<0.001$ \\
\hline Yes & 33 & 34.7 & 9 & 11.8 & 42 & 24.6 & \\
\hline Work affected & & & & & & & \\
\hline No & 40 & 42.1 & 53 & 69.7 & 93 & 54.4 & $<0.0001$ \\
\hline Yes & 44 & 46.3 & 9 & 11.8 & 53 & 31.0 & \\
\hline Don't know, no response, not applicable & 11 & 11.6 & 14 & 18.4 & 25 & 14.6 & \\
\hline Leisure affected & & & & & & & \\
\hline No & 53 & 55.8 & 73 & 96.1 & 126 & 73.7 & $<0.0001$ \\
\hline Yes & 42 & 44.2 & 3 & 3.9 & 45 & 26.3 & \\
\hline Impact on emotional and behavi & status & & & & & & \\
\hline Post-traumatic stress & & & & & & & \\
\hline No & 75 & 78.9 & 71 & 93.4 & 146 & 85.4 & $<0.01$ \\
\hline Yes & 20 & 21.1 & 5 & 6.6 & 25 & 14.6 & \\
\hline Feeling low & & & & & & & \\
\hline Not at all or Not more than usual & 46 & 48.4 & 68 & 89.5 & 114 & 66.7 & $<0.0001$ \\
\hline A little more or Much more than usual & 49 & 51.6 & 8 & 10.5 & 57 & 33.3 & \\
\hline Feeling nervous & & & & & & & \\
\hline Not at all & 37 & 38.9 & 51 & 67.1 & 88 & 51.5 & $<0.001$ \\
\hline A little, sometimes & 38 & 40.0 & 19 & 25.0 & 57 & 33.3 & \\
\hline Often, very often & 20 & 21.1 & 6 & 7.9 & 26 & 15.2 & \\
\hline Negative feelings (despair, depression, etc.) & & & & & 0 & 0.0 & \\
\hline Not at all & 18 & 18.9 & 22 & 28.9 & 40 & 23.4 & 0.02 \\
\hline Sometimes & 48 & 50.5 & 44 & 57.9 & 92 & 53.8 & \\
\hline Often, always & 29 & 30.5 & 10 & 13.2 & 39 & 22.8 & \\
\hline
\end{tabular}

family member) or psychological history or PTSD at 1 year on multivariate analysis, although these factors had been related to persistent pain on univariate analysis.

\section{Discussion}

The study sought to assess the impact of various factors on health status at 1 year after whiplash injury and to elucidate the role of pain. Half of the victims had not fully recovered health status. The main factor associated with failure to recover was pain; another was not having been responsible for the accident. The main factors related to pain were gender and non-responsibility in the accident.

The only sociodemographic factor contributing to the prediction of poor recovery and persistent pain was gender. An association, however slight [18], between female gender and the consequences of whiplash injury has frequently been reported [4] [19] [20]. The effect of gender on the risk of subsequent disability is a matter of debate: Walton et al. [8] found elevated risk in women in the 11 cohorts they studied, in contrast to ScholtenPeeters' review of the literature [5]. Excess risk was recently found by Merrick and Stålnacke [21], who found 
Table 4. Factors associated with non-recovery of health status at 1 year (modified Poisson regression).

\begin{tabular}{|c|c|c|c|c|c|c|c|c|}
\hline & \multicolumn{2}{|c|}{ Non-recovery $(n=95)$} & \multicolumn{2}{|c|}{ Recovery $(n=76)$} & \multicolumn{2}{|c|}{ Model 1} & \multicolumn{2}{|c|}{ Model 2} \\
\hline & $\mathrm{n}$ & $\%$ & $\mathrm{n}$ & $\%$ & Adjusted RR & P-value & Adjusted RR & P-value \\
\hline \multicolumn{9}{|l|}{ Adjustment variables } \\
\hline Whiplash grade & & & & & & NS & & NS \\
\hline grade 1 & 32 & 33.7 & 30 & 39.5 & ref & & ref & \\
\hline grade 2 & 63 & 66.3 & 46 & 60.5 & $1.1[0.8-1.4]$ & & $1.0[0.8-1.3]$ & \\
\hline Gender & & & & & & $<0.04$ & & NS \\
\hline Male & 30 & 31.6 & 34 & 44.7 & ref & & ref & \\
\hline Female & 65 & 68.4 & 42 & 55.3 & $1.3[1.0-1.8]$ & & $1.2[1.0-1.5]$ & \\
\hline Age & & & & & & $<0.05$ & & NS \\
\hline $16-24$ yrs & 22 & 23.2 & 32 & 42.1 & ref & & ref & \\
\hline $25-34$ yrs & 32 & 33.7 & 14 & 18.4 & $1.6[1.1-2.3]$ & & $1.3[0.9-1.7]$ & \\
\hline $35-44$ yrs & 25 & 26.3 & 15 & 19.7 & $1.4[0.9-2.0]$ & & $1.1[0.8-1.4]$ & \\
\hline$>=45 \mathrm{yrs}$ & 16 & 16.8 & 15 & 19.7 & $1.2[0.7-1.9]$ & & $0.9[0.6-1.3]$ & \\
\hline \multicolumn{9}{|l|}{ Inclusion variables } \\
\hline Pre-accident event: birth & & & & & & $<0.01$ & & NS \\
\hline No & 68 & 71.6 & 65 & 85.5 & ref & & ref & \\
\hline Yes & 27 & 28.4 & 11 & 14.5 & $1.6[1.2-2.1]$ & & $1.1[0.9-1.4]$ & \\
\hline $\begin{array}{l}\text { Pre-accident event: } \\
\text { Death, hospital admission }\end{array}$ & & & & & & $<0.04$ & & NS \\
\hline No & 56 & 58.9 & 56 & 73.7 & ref & & ref & \\
\hline Yes & 39 & 41.1 & 20 & 26.3 & $1.3[1.0-1.7]$ & & $1.2[0.9-1.4]$ & \\
\hline Responsibility & & & & & & $<0.03$ & & NS \\
\hline No & 79 & 83.2 & 49 & 64.5 & ref & & ref & \\
\hline Yes & 16 & 16.8 & 27 & 35.5 & $0.7[0.4-1.0]$ & & $0.8[0.6-1.1]$ & \\
\hline \multicolumn{9}{|l|}{ 1-year variables } \\
\hline Memory disorder & & & & & - & & & $<0.01$ \\
\hline Not at all or Not more than usual & 63 & 66.3 & 73 & 96.1 & & & ref & \\
\hline A little or Much more than usual & 32 & 33.7 & 3 & 3.9 & & & $1.5[1.2-1.8]$ & \\
\hline Disabling pain & & & & & - & & & $<0.0001$ \\
\hline No & 19 & 20.0 & 66 & 86.8 & & & ref & \\
\hline Yes & 75 & 78.9 & 10 & 13.2 & & & $3.3[2.2-5.1]$ & \\
\hline
\end{tabular}

females to be over-represented in the poor recovery group. The main reason for such a sex difference could bephysiological [22]: cervical spine stability may be greater in males, due to their anatomically larger vertebrae [23] or due to the relation between muscle stiffness and hormones [24]; conversely, Vibert [25] observed, in experiments in which seated participants were subjected to low-amplitude high-jerk linear acceleration impulses, that "stiff" participants (more likely females) showed little head movement, whereas the "floppy" participants showed a large head rotation in the direction opposite the movement: this could signify that females have greater 
Table 5. Factors associated with persistent pain at 1 year (modified Poisson regression).

\begin{tabular}{|c|c|c|c|c|c|c|c|c|}
\hline & \multicolumn{2}{|c|}{ Persistent pain $(n=86)$} & \multicolumn{2}{|c|}{ No pain $(n=85)$} & \multicolumn{2}{|c|}{ Model 1} & \multicolumn{2}{|c|}{ Model 2} \\
\hline & $\mathrm{n}$ & $\%$ & $\mathrm{n}$ & $\%$ & Adjusted RR & $\mathrm{p}$-value & Adjusted RR & p-value \\
\hline \multicolumn{9}{|c|}{ Adjustment variables } \\
\hline Whiplash grade & & & & & & NS & & NS \\
\hline grade 1 & 27 & 31.4 & 35 & 41.2 & ref & & ref & \\
\hline grade 2 & 59 & 68.6 & 50 & 58.8 & $1.3[0.9-1.7]$ & & $1.0[0.8-1.4]$ & \\
\hline Gender & & & & & & NS & & 0.003 \\
\hline Male & 27 & 31.4 & 37 & 43.5 & ref & & ref & \\
\hline Female & 59 & 68.6 & 48 & 56.5 & $1.3[1.0-1.9]$ & & $1.5[1.1-2.1]$ & \\
\hline Age & & & & & & NS & & NS \\
\hline $16-24$ yrs & 20 & 23.3 & 34 & 40.0 & ref & & ref & \\
\hline $25-34$ yrs & 25 & 29.1 & 21 & 24.7 & $1.4[0.9-2.1]$ & & $0.9[0.6-1.3]$ & \\
\hline $35-44$ yrs & 24 & 27.9 & 16 & 18.8 & $1.5[1.0-2.3]$ & & $0.8[0.5-1.3]$ & \\
\hline$>=45 \mathrm{yrs}$ & 17 & 19.8 & 14 & 16.5 & $1.4[0.9-2.3]$ & & $0.9[0.6-1.4]$ & \\
\hline \multicolumn{9}{|l|}{ Inclusion variables } \\
\hline Responsibility & & & & & & $<0.0 .02$ & & 0.02 \\
\hline No & 74 & 86.0 & 54 & 63.5 & ref & & ref & \\
\hline Yes & 12 & 14.0 & 31 & 36.5 & $0.5[0.3-0.9]$ & & $0.6[0.4-1.0]$ & \\
\hline Pre-accident event: birth & & & & & & $<0.02$ & & NS \\
\hline No & 60 & 62.5 & 73 & 96.1 & ref & & ref & \\
\hline Yes & 13 & 15.1 & 3 & 3.5 & $1.6[1.2-2.2]$ & & $1.4[1.0-1.9]$ & \\
\hline \multicolumn{9}{|c|}{ 1-year variables } \\
\hline Leisure affected & & & & & - & & & $<0.001$ \\
\hline No & 45 & 52.3 & 81 & 95.3 & & & ref & \\
\hline Yes & 41 & 47.7 & 4 & 4.7 & & & $1.7[1.3-2.3]$ & \\
\hline Medical drug consumption & & & & & - & & & 0.001 \\
\hline No & 43 & 50.0 & 82 & 96.5 & & & ref & \\
\hline Yes & 43 & 50.0 & 3 & 3.5 & & & $1.7[1.3-2.4]$ & \\
\hline Work affected & & & & & - & & & $<0.01$ \\
\hline No & 35 & 40.7 & 58 & 68.2 & & & ref & \\
\hline Yes & 43 & 50.0 & 10 & 11.8 & & & $1.6[1.2-2.1]$ & \\
\hline $\begin{array}{c}\text { Don't know, no response, } \\
\text { not applicable }\end{array}$ & 8 & 9.3 & 17 & 20.0 & & & - & \\
\hline
\end{tabular}

muscle control, and perhaps more lesions induced by violent muscle tension, than males. Several studies [8] [19] [26] highlighted a risk of poorer recovery in subjects with lower educational level. The present results, however found no such correlation.

All studies agree that crash-related factors, with the exception of seat-belt use, are irrelevant to recovery and persistent pain [3] [6] [8] [27] [28]. The present study partially replicated these findings, in that type of road- 
user, position in the vehicle and impact direction were not predictive of residual symptoms at 1 year, on which only the type of antagonist had an effect. Greater initial whiplash injury severity was reported to be associated with significantly slower recovery [4] [26]; this finding was not so clear in our study, initial severity factors contributing only slightly to prognosis for recovery.

Previous studies reported contradictory conclusions as to the prognostic significance of psychological factors, but generally found them to be predictive of recovery rate [28]. Richter et al. [27] considered psychosocial factors more relevant than lesion severity in terms of symptom duration. Pain seems to be associated with and increased by anxiety and depression [18] [29]-[31]. The present study, on the other hand, found no correlation with psychological factors: while associated with risk of non-recovery on univariate analysis, none of these factors were conserved in the final explanatory model. Notably, "pure" whiplash victims did not suffer PTSD more frequently than the cohort as a whole [32].

The present results showed a lower risk of persistent pain in drivers responsible for their accident. This may be understood in the light of studies [4] [33] [34] showing specific patterns of functional recovery in subjects claiming compensation. A feeling of injustice often leads victims to sue; litigation proceeds very slowly, keeping the victim in a waiting situation that does not favor recovery. A physical hypothesis, however, may also be considered: in an accident for which they bear responsibility, drivers have seen the crash coming and contracted their muscles to prepare for the shock, whereas innocent victims are taken by surprise, especially in case of rear impact; this hypothesis is borne out by the findings of Fice et al. [35] that muscle activation at the moment of the shock can protect the capsular ligament. Consequently, the influence of emotional state on prompt recovery may be modulated by pain [27], which, however, is the main predictive factor of delayed recovery after trauma, rather than the inverse.

Pain is related to impact on daily life. The specific association with memory disorder is in agreement with an American study [36] which found greater neck disability in whiplash associated with disturbed memory.

The present study has several strong points. The ESPARR cohort is a prospective road-accident victim cohort, representative of accidents in a precise geographic area for which a Registry of road accident injuries has been operating since 1995 [13] [37]. Comparison of whiplash victims in the ESPARR cohort and those included in the Registry but not in ESPARR showed no selection bias. The study population was representative of whiplash victims in general, providing a picture not focused on any particular type of road user or accident parameter or exclusively on victims consulting for persistent pain.

The various questionnaires did not specifically target whiplash, as the ESPARR cohort includes all kinds of lesion; this avoided the subjectivity bias phenomenon whereby patients report more pain in regions of interest to the investigator.

Several limitations, however, remain. As ESPARR concerns road accident victims in general, the questionnaire used at inclusion did not comprise specific whiplash assessment tools such as the Neck Disability Index (NDI); this prevented assessment of initial pain as the prime predictive factor, although severity assessment was based on emergency examination and X-ray data, which indirectly indicated shock intensity. Furthermore, the initial classification of lesions in terms of AIS was based on emergency reports by a number of physicians: this may have led to variations in diagnosis and in classification between the two severity grades. Despite a fairly large sample, power was lacking for some analyses.

A further limitation may lie in the use of subjective variables for 1-year health assessment. However, self-assessment of health recovery has been shown to be reliable for epidemiological purposes [38]. Moreover, the pain item was formulated in terms of pain that is disabling in everyday life, in the spirit of the Quebec Task Force.

\section{Conclusion}

In conclusion, the present results obtained from a representative population of whiplash victims extend our understanding of whiplash injury. Residual pain is the fundamental factor causing whiplash victims to feel that they have not recovered good health. Our findings suggest that this may be bound up with physical factors.

\section{Acknowledgements}

The authors are grateful to the victims for their cooperation in data collection. The authors would like to thank all those who assisted in carrying out this study: Nadia Baguena, Jean Yves Bar, Amélie Boulanger, Élodie Paquelet, Stuart Nash and Véronique Sotton for collecting the data; Irène Vergnes for organizing the databases; 
Anne-Marie Bigot, Nathalie Demangel and Geneviève Boissier for subject database management; Blandine Gadegbeku, Amina NDiaye and the Association for the Rhône Road Trauma Registry (ARVAC) for their help in collecting and providing medical data; the Scientific Committee (Daniel Floret, François Chapuis, Jean Michel Mazaux, Jean Louis Martin, Jacques Gaucher and Étienne Javouhey); Dominique Boisson and Jacques Luauté for their participation in the ESPARR scientific management team; and all the hospital staff who accepted the interviewers' presence and referred victims. Thanks to P.P. Vidal and other members of the whiplash consortium of the Road Safety Foundation program for the discussions we had together.

Special thanks to Iain McGill for comments and manuscript editing.

\section{Funding Sources}

We acknowledge funding from the French Ministry of Equipment, Transport, Housing, Tourism and the Sea (Predit 3 Program, "New Knowledge in the Field of Road Safety": № SU0400066) and from the French Ministry of Health (PHRC 2003 Program, PHRC-N03; PHRC 2005 Program, PHRC-N051) and from the Road Safety Foundation (2010/MP/01/).

The individual authors have no competing interests to declare.

Informed consent was obtained from all subjects.

\section{References}

[1] Spitzer, W.O., Skovron, M.L., Salmi, L.R., Cassidy, J.D., Duranceau, J., Suissa, S. and Zeiss, E. (1995) Scientific Monograph of the Quebec Task Force on Whiplash-Associated Disorders: Redefining "Whiplash” and Its Management. Spine, 20, 1S-73S.

[2] AAAM, Association for the Advancement of Automotive Medicine (1990) The Abbreviated Injury Scale-1990 Revision (AIS-90). Des Plaines.

[3] Richter, M., Otte, D., Pohlemann, T., Krettek, C. and Blauth, M. (2000) Whiplash-Type Neck Distortion in Restrained Car Drivers: Frequency, Causes and Long-Term Results. European Spine Journal, 9, 109-117. http://dx.doi.org/10.1007/s005860050220

[4] Côté, P., Cassidy, J.D., Carroll, L.J., Frank, J.W. and Bombardier, C. (2001) A Systematic Review of the Prognosis of Acute Whiplash and a New Conceptual Framework to Synthesize the Literature. Spine, 26, E445-E458. http://dx.doi.org/10.1097/00007632-200110010-00020

[5] Scholten-Peeters, G.M., Verhagen, P., Bekkering, G.E., Van Der Windt, D.A., Barnsley, L., Oostendorp, R.A.B. and Hendriks, E.J. (2003) Prognostic Factors of Whiplash-Associated Discorders: A Systematic Review of Prospective Cohort Studies. Pain, 104, 303-322. http://dx.doi.org/10.1016/S0304-3959(03)00050-2

[6] Williams, M., Williamson, E., Gates, S., Lamb, S. and Cooke, M. (2007) A Systematic Literature Review of Physical Prognostic Factors for the Development of Late Whiplash Syndrome. Spine, 32, E764-E780.

http://dx.doi.org/10.1097/BRS.0b013e31815b6565

[7] Williamson, E., Williams, M., Gates, S. and Lamb, S.E. (2008) A Systematic Literature Review of Psychological Factors and the Development of Late Whiplash Syndrome. Pain, 135, 20-30. http://dx.doi.org/10.1016/j.pain.2007.04.035

[8] Walton, D., Pretty, J., Macdermid, J. and Teasell, R. (2009) Risk Factors for Persistent Problems Following Whiplash Injury: Results of a Systematic Review and Meta-Analysis. Journal of Orthopaedic \& Sports Physical Therapy, 39, 334-350. http://dx.doi.org/10.2519/jospt.2009.2765

[9] Carroll, L.J., Holm, L.W., Hogg-Johnson, S., et al. (2008) Course and Prognostic Factors for Neck Pain in WhiplashAssociated Disorders (WAD): Results of the Bone and Joint Decade 2000-2010 Task Force on Neck Pain and Its Associated Disorders. Spine, 33, S83-S92. http://dx.doi.org/10.1097/BRS.0b013e3181643eb8

[10] Revel, M. (2003) Whiplash Injury of the Neck from Concepts to Facts. Annales de Réadaptation et de Médecine Physique, 46, 158-170. http://dx.doi.org/10.1016/S0168-6054(03)00053-9

[11] Binder, A. (2007) The Diagnosis and Treatment of Nonspecific Neck Pain and Whiplash. Eura Medicophysics, 43, 7989.

[12] Hours, M., Bernard, M., Charnay, P., et al. (2010) Functional Outcome after Road-Crash Injury: Description of the ESPARR Victims Cohort and 6-Month Follow-Up Results. Accident Analysis \& Prevention, 42, 412-421. http://dx.doi.org/10.1016/j.aap.2009.09.002

[13] Laumon, B., Martin, J.L., Collet, P., Chiron, M., Verney, M.P., Ndiaye, A. and Vergnes, I. (1997) A French Road Accident Trauma Registry : First Results. 41st AAAM Conference, 1997, AAAM, Orlando.

[14] Charnay, P. and Laumon, B. (2002) Le registre des victimes d’accidents de la circulation routière du Rhône: Modalités 
de mise en place, de recueil, d'informatisation et de gestion. Rapport UMRESTTE, 1-119.

[15] The Whoqol Group (1998) Development of the World Health Organization WHOQOL-BREF Quality of Life Assessment. Psychological Medicine, 28, 551-558. http://dx.doi.org/10.1017/S0033291798006667

[16] Weathers, F.W., Litz, B.T., Herman, D.S., Huska, J.A. and Keane, T.M. (1993) The PTSD Checklist: Reliability, Validity and Diagnostic Utility. IXth Annual Meeting of the International Society for Traumatic Stress Studies, 1993, San Antonio.

[17] Ventureyra, V., Yao, S.-N., Cottraux, J., Note, I. and De Mey-Guillard, C. (2002) The Validation of the Posttraumatic Stress Disorder Checklist Scale in Posttraumatic Stress Disorder and Nonclinical Subjects. Psychotherapy Psychosom, 71, 47-53. http://dx.doi.org/10.1159/000049343

[18] Sullivan, M.J., Stanish, W., Sullivan, M.E. and Tripp, D. (2002) Differential Predictors of Pain and Disability in Patients with Whiplash Injuries. Pain Research Managing, 7, 68-74.

[19] Hendriks, E.J.M., Scholten-Peeters, G.G., Van Der Windt, D.A.W.M., Neeleman-Van Der Steen, C.W.M., Oostendorp, R.A.B. and Verhagen, A.P. (2005) Prognostic Factors for Poor Recovery in Acute Whiplash Patients. Pain, 114, 408416. http://dx.doi.org/10.1016/j.pain.2005.01.006

[20] Carstensen, T., Frostholm, L., Oernboel, E., Kongsted, A., Kasch, H., Jensen, T. and Fink, P. (2012) Are There Gender Differences in Coping with Neck Pain Following Acute Whiplash Trauma? A 12-Month Follow-Up Study. European Journal of Pain, 16, 49-60. http://dx.doi.org/10.1016/j.ejpain.2011.06.002

[21] Merrick, D. and Stålnacke, B.M. (2010) Five Years Post Whiplash Injury: Symptoms and Psychological Factors in Recovered versus Non-Recovered. BMC Research Notes, 3, 190. http://dx.doi.org/10.1186/1756-0500-3-190

[22] Stemper, B., Derosia, J., Yogananan, N., Pintar, F., Shender, B. and Paskoff, G. (2009) Gender Dependent Cervical Spine Anatomical Differences in Size-Matched Volunteers-Biomed 2009. Biomedical Sciences Instrumentation, 45, 149154.

[23] Stemper, B.D., Yoganandan, N., Pintar, F.A., Maiman, D.J., Meyer, M.A., DeRosia, J., Shender, B.S. and Paskoff, G. (2008) Anatomical Gender Differences in Cervical Vertebrae of Size-Matched Volunteers. Spine, 33, E44-E49. http://dx.doi.org/10.1097/BRS.0b013e318160462a

[24] Bell, D.R., Blackburn, J.T., Norcorss, M.F., Ondrak, K.S., Hudson, J.D., Hackney, A.C. and Padua, D.A. (2012) Estrogen and Muscle Stiffness Have a Negative Relationship in Females. Knee Surgery, Sports Traumatology, Arthroscopy, 20, 361-367. http://dx.doi.org/10.1007/s00167-011-1577-y

[25] Vibert, N., Hoang, T., Gilchrist, D.P., MacDougall, H.G., Burgess, A.M., Roberts, R.D., Vidal, P.P. and Curthoys, I.S. (2006) Psychophysiological Correlates of the Inter-Individual Variability of Head Movement Control in Seated Humans. Gait \& Posture, 23, 355-363. http://dx.doi.org/10.1016/j.gaitpost.2005.04.006

[26] Sterner, Y., Toolanen, G., Gerdle, B. and Hildingsson, C. (2003) The Incidence of Whiplash Trauma and the Effects of Different Factors on Recovery. Journal of Spinal Disorders \& Techniques, 16, 195-199. http://dx.doi.org/10.1097/00024720-200304000-00013

[27] Richter, M., Ferrari, R., Otte, D., Kuensebeck, H.W., Blauth, M. and Krettek, C. (2004) Correlation of Clinical Findings, Collision Parameters, and Psychological Factors in the Outcome of Whiplash Associated Disorders. Journal of Neurology, Neurosurgery \& Psychiatry, 75, 758-764. http://dx.doi.org/10.1136/jnnp.2003.026963

[28] Carroll, L.J., Holm, L.W., Hogg-Johnson, S., et al. (2009) Course and Prognostic Factors for Neck Pain in WhiplashAssociated Disorders (WAD). Results of the Bone and Joint Decade 2000-2010 Task Force on Neck Pain and Its Associated Disorders. Journal of Manipulative and Physiological Therapeutics, 32, S97-S107. http://dx.doi.org/10.1016/j.jmpt.2008.11.014

[29] Ferrari, R. (2004) The Clinical Relevance of Symptom Amplification. Pain, 107, 276-277. http://dx.doi.org/10.1016/j.pain.2003.11.014

[30] Buitenhuis, J., De Jong, P., Jaspers, J. and Groothoff, J. (2008) Catastrophizing and Causal Beliefs in Whiplash. Spine (Phila Pa 1976), 33, 2427-2433.

[31] Börsbo, B., Peolsson, M. and Gerdle, B. (2008) Catastrophizing, Depression, and Pain: Correlation with and Influence on Quality of Life and Health-A Study of Chronic Whiplash-Associated Disorders. Journal of Rehabilitation Medicine, 40, 562-569. http://www.medicaljournals.se/jrm/content/?doi=10.2340/16501977-0207

[32] Chossegros, L., Hours, M., Charnay, P., et al. (2011) Predictive Factors of Chronic Post-Traumatic Stress Disorder 6 Months after a Road Traffic Accident. Accident Analysis \& Prevention, 43, 471-477. http://dx.doi.org/10.1016/j.aap.2010.10.004

[33] Sterling, M., Hendrikz, J. and Kenardy, J. (2010) Compensation Claim Lodgement and Health Outcome Developmental Trajectories Following Whiplash Injury: A Prospective Study. Pain, 150, 22-28. http://dx.doi.org/10.1016/j.pain.2010.02.013 
[34] Ferrari, R. and Russell, A.S. (2001) Why Blame Is a Factor in Recovery from Whiplash Injury. Medical Hypotheses, 56, 372-375. http://dx.doi.org/10.1054/mehy.2000.1215

[35] Fice, J.B., Cronin, D.S. and Panzer, M.B. (2011) Cervical Spine Model to Predict Capsular Ligament Response in Rear Impact. Annals of Biomedical Engineering, 39, 2152-2162. http://dx.doi.org/10.1007/s10439-011-0315-4

[36] Robinson, J.P., Burwinkle, T. and Turk, D.C. (2007) Perceived and Actual Memory, Concentration, and Attention Problems after Whiplash-Associated Disorders (Grades I and II): Prevalence and Predictors. Archives of Physical Medicine and Rehabilitation, 88, 774-779. http://dx.doi.org/10.1016/j.apmr.2007.03.004

[37] Amoros, E., Martin, J.L. and Laumon, B. (2006) Under-Reporting of Road Crash Casualties in France. Accident Analysis \& Prevention, 38, 627-635. http://dx.doi.org/10.1016/j.aap.2005.11.006

[38] Ngo, T., Stupar, M., Côté, P., Boyle, E. and Shearer, H. (2010) A Study of the Test-Retest Reliability of the Self-Perceived General Recovery and Self-Perceived Change in Neck Pain Questions in Patients with Recent Whiplash-Associated Disorders. European Spine Journal, 19, 957-962. http://dx.doi.org/10.1007/s00586-010-1289-X 